Journal of Non-Newtonian Fluid Mechanic8, 6 (1979) 111-125

(C) Elsevier Scientific Publishing Company, Amsterdam - Printed in The Netherlands

\title{
ON THE SIMULTANEOUS ELONGATION AND INFLATION OF A TUBULAR MEMBRANE OF BKZ FLUID
}

\section{ALAN WINEMAN}

Department of Applied Mechanics and Engineering Sciences, The University of Michigan, Ann Arbor, Michigan 48109 (U.S.A.)

(Received April 10, 1978; accepted in revised form February 28, 1979)

\section{Summary}

This work considers a viscoelastic fluid membrane which is initially tubular and bonded at each end to a rigid circular disc. The membrane is subjected to prescribed elongational and internal pressure histories causing it to undergo quasi-static axisymmetric deformation. This example is intended to simulate an experiment which has been recently proposed for the determination of constitutive properties for viscoelastic fluids as well as some polymer sheet forming process.

The constitutive equation is presumed to be of integral type. The formulation of the problem leads to a basic system of equations which is intended for numerical solution. It has the structure of a two-point boundary value problem for a system ordinary differential equations at each time. The formulation has the advantage that the equations do not have to be rederived if the constitutive equation is changed. A change in the sub-program for computing stress from stretch history is all that is needed.

A numerical method of solution is presented. In a numerical example, the material is taken to be polyisobutylene, modeled as a BKZ fluid.

\section{Introduction}

Polymer forming processes such as drape or vacuum forming subject polymer sheets to large stretching type deformations. If the sheet is sufficiently thin and the variation of stretching sufficiently gradual, it can be regarded as a membrane. This implies that bending effects are negligible compared with stretching effects and that the variation of stresses through the sheet thickness can be neglected. It also permits the process to be studied in the con- 
text of the theory of large deformations of membranes [1].

The polymer materials used in such processes can be regarded as viscoelastic fluids. Consistent with the membrane nature of these processes is the determination of constitutive equations for such materials under conditions of planar stretching. A number of experiments have been devised for this purpose. The simplest such experiment, conceptually, is to subject a specimen to homogeneous unequal biaxial stretch histories. However, it is difficult to develop a mechanism for applying independent stretch histories in two perpendicular directions. A rheological experiment which avoids this difficulty is the inflation of a thin circular sheet by pressurizing one side [2]. The sheet, clamped at its outer boundary, undergoes an axially symmetric deformation. At each time it forms a sphere-like membranous cap. The polar region, which undergoes a locally homogeneous equal biaxial stretch history, is a source of data. Although useful, such data is inadequate for a complete characterization of material response.

A more promising experiment has recently been developed by Chung and Stevenson [3]. An initially circular viscoelastic tube, bonded at its ends to rigid plates, is subjected to simultaneous extension and internal pressure. The deformation is symmetric not only about a central axis, but also with respect to a plane which is perpendicular to this axis and midway between the end plates. This central region is cylindrical and has an essentially homogeneous unequal biaxial stretch ratio distribution. By adjusting the internal pressure and either the extensional force or elongation histories, the stretch ratio histories can be varied. In the analysis, the tube is not necessarily thin-walled and only the cylindrical region is considered. It is shown that sufficient constitutive data can be obtained to characterize the materials.

The purpose of the present paper is to provide a means for determining the deformation history of the complete tube under the assumption that it can be regarded as a membrane. The approach will be applicable for an arbitrary choice of constitutive equation of integral type. There are several reasons for developing such a procedure. Because of the end constraints on the tube, the deformation will be non-homogeneous. By solving the problem for the complete tube, an estimate can be made of the size of the region in which the deformation is approximately homogeneous. Secondly, suppose a specific form for a constitutive equation is developed from experiments. Using this, the profile history of the deformed membrane can be computed. It can then be compared with a measured profile history in order to assess the accuracy of the constitutive equation. Finally, this study presents an opportunity for the continued development of numerical procedures which could be of use in studying actual polymer forming processes.

The problem is defined and the governing equations are presented in Section 2 . In Section 3, these equations are reduced to the basic system which is intended for numerical solution. This formulation is such that the system of equations is unaffected by a change in constitutive equation. A change in the latter requires only a change in the subprogram for computing stress from 
deformation history. The general numerical procedure reduces the problem to solving a system of ordinary differential equations at each time step. In previous work $[4,5]$, boundary conditions required determining a single quan. tity at one end of an interval in order to satisfy one condition at the other end. In this problem, two conditions must be determined at one end to satisfy two conditions at the other. These numerical procedures are discussed in Section 4. Although the procedure is valid for any constitutive equation, it is illustrated for a particular choice. One such choice is presented in Section 2. Results for an example are presented in Section 5 . It was found that the numerical procedure solved the two point boundary value problem successfully at a fixed time $t_{n}$. However, the method for determining the initial estimate for the end conditions at the time $t_{n+1}$ needs to be improved. This is also discussed in Section 5.

\section{Formulation}

The undeformed membrane has a circular cylindrical midsurface of radius $a$ and length $2 L_{0}$. Its wall thickness is initially uniform and is denoted by $h_{0}$. The ends are bonded to rigid circular discs of radius $a$. These are considered attached to a loading device which applies a prescribed internal pressure history $p(t)$ and either a prescribed extensional force history $F(t)$ or an elongation history $L(t)$.

As indicated in Fig. 1, the deformation is referred to a cylindrical polar

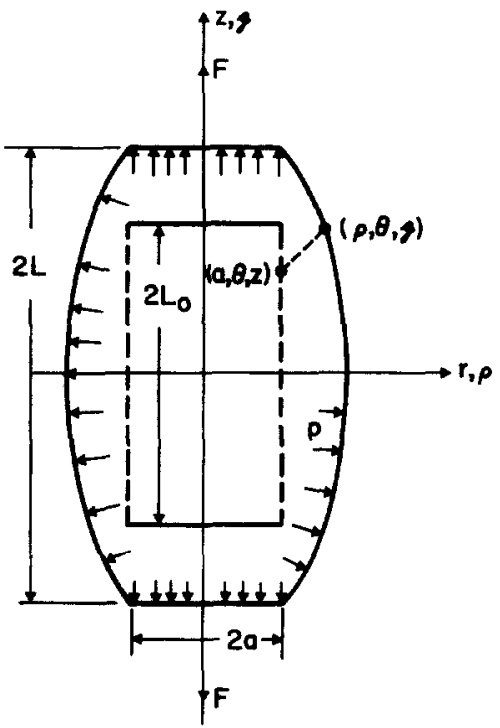

Fig. 1. Undeformed and deformed configurations of the tubular membrane. Dashed lines denote the undeformed state. 
coordinate system with the polar-axis along the tube center line and the origin at mid height. The membrane is assumed to undergo quasi-static motion, with a typical particle initially at $(a, \theta, z)$ moving to $(\rho(z, t), \theta, \zeta(z, t))$ at time $t>0$. In view of the axisymmetry, the principal directions of stretch and stress are known at each time $t$. At each particle these are in the meridional (1) and circumferential (2) directions and also normal (3) to the surface. The principal stretch ratios in these directions are given by, respectively,

$\lambda_{1}=\left[\left(\frac{\partial \rho}{\partial z}\right)^{2}+\left(\frac{\partial \zeta}{\partial z}\right)^{2}\right]^{1 / 2}, \quad \lambda_{2}=\frac{\rho}{a}, \quad \lambda_{3}=\frac{1}{\lambda_{1} \lambda_{2}}$,

where $\lambda_{3}$ is determined by the assumption of incompressibility. It is convenient to introduce the following associated kinematic variable:

$\eta=\partial \rho / \partial z$.

If $\sigma_{\alpha}$ denotes a principal stress, its stress resultant per unit length of circumferential or meridional line in the membrane surface is $T_{\alpha}=h_{0} \lambda_{3} \sigma_{\alpha},(\alpha=1,2)$. The force balance equations appropriate to the study of large axially symmetric deformations of membranes are presented in [1, Section 4.11]. In the meridional and normal directions relative to the membrane at time $t$, these are, respectively,

$\frac{\partial T_{1}}{\partial z}+\frac{\eta\left(T_{1}-T_{2}\right)}{a \lambda_{2}}=0$,

$\kappa_{1} T_{1}+\kappa_{2} T_{2}=p$.

In (2.4), $\kappa_{1}$ and $\kappa_{2}$ are principal curvatures given by

$$
\kappa_{1}=\frac{\left(\eta\left(\partial \lambda_{1} / \partial z\right)-\lambda_{1}(\partial \eta / \partial z)\right)}{\lambda_{1}^{2}\left[\lambda_{1}^{2}-\eta^{2}\right]^{1 / 2}}, \quad \kappa_{2}=\frac{\left[\lambda_{1}^{2}-\eta^{2}\right]^{1 / 2}}{a \lambda_{1} \lambda_{2}}
$$

Equations (2.3)-(2.5) have been expressed in terms of the kinematic quantities $\lambda_{1}, \lambda_{2}$ and $\eta$ instead of $\rho$ and $\zeta$ for the reasons discussed in [4]. Note that these equations have been transformed to be in terms of the independent variable $z$ which serves as a particle label in the initial configuration.

As in the case of the problem of the deformation of an initially plane viscoelastic membrane by lateral pressure [4], eqns. (2.3)-(2.5) can be simplified. Recall the Codazzi relation satisfied by the principal curvatures, $[1, p .151]$,

$\frac{\partial}{\partial \rho}\left(\rho \kappa_{2}\right)=\kappa_{1}$.

Let $F$ denote the force on an end-plate. It can be shown, using (2.6), that (2.3) and (2.4) can be replaced, at each time $t$, by (2.3) and

$2 \pi \rho^{2} \kappa_{2} T_{1}=p \pi \rho^{2}+F, \quad 0 \leqslant z \leqslant L_{0}$.

Since, as (2.5) indicates, this equation does not contain spatial derivatives 


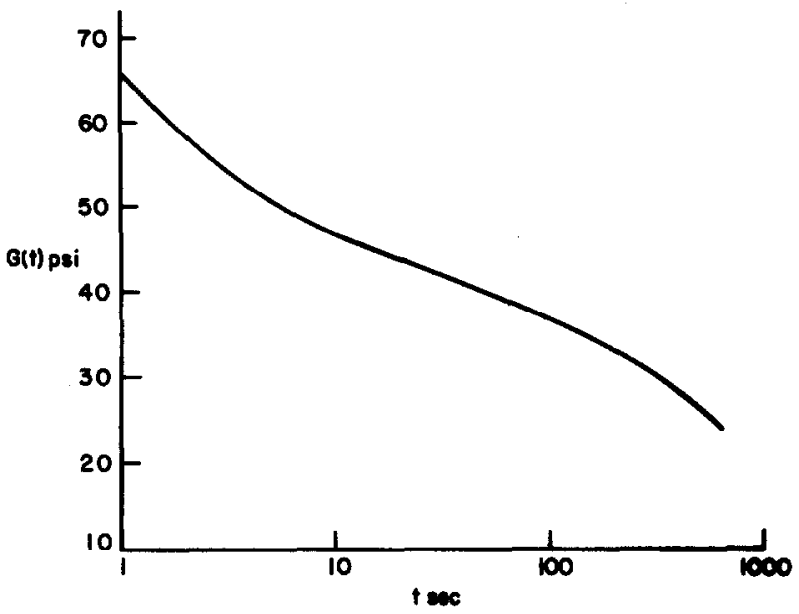

Fig. 2. Relaxation Function $G(t)$ for the assumed model.

of $\lambda_{1}, \lambda_{2}$ or $\eta$ it can be regarded as a first integration of the system of equations.

The membrane material is assumed to be an incompressible homogeneous viscoelastic fluid. The solution procedure presented later can be applied for any type of constitutive equation in principle. However, for purposes of illustration, it is assumed that the fluid can be modeled by a non-linear single integral constitutive equation. Let $\lambda(s)$ denote the pair $\left(\lambda_{1}(s), \lambda_{2}(s)\right)$. Then the models under consideration have the form

$\sigma_{\alpha}(t)=A_{\alpha}[\lambda(t), t]+\int_{0}^{t} B_{\alpha}[\lambda(t) / \lambda(s), t-s] \mathrm{d} s, \quad(\alpha=1,2)$.

Functions $A_{\alpha}$ and $B_{\alpha}$ depend on the choice of material.

One material which has been used in the membrane inflation experiment and other thin sheet forming studies is polyisobutylene [2]. A specific constitutive equation of form (2.8) for polyisobutylene which can be used for sheet problems does not appear to be available. For the present purposes, one was constructed using the BKZ [6] model, for which some experimental data is available. With respect to principal directions, it has the following form:

$$
\begin{aligned}
\sigma_{\alpha}(t)= & 2\left(\lambda_{\alpha}^{2}(t)-\lambda_{3}^{2}(t)\right)\left[1+\frac{4.5\left(1+\lambda_{\beta}^{2}(t)\right)}{I_{1}(t, 0)+I_{2}(t, 0)+3}\right] G(t) \\
& -2 \int_{0}^{t}\left[\frac{\lambda_{\alpha}^{2}(t)}{\lambda_{\alpha}^{2}(s)}-\frac{\lambda_{3}^{2}(t)}{\lambda_{3}^{2}(s)}\right]\left[1+\frac{4.5}{I_{1}(t, s)+I_{2}(t, s)+3}\left(1+\frac{\lambda_{\beta}^{2}(t)}{\lambda_{\beta}^{2}(s)}\right)\right] \dot{G}(t-s) \mathrm{d} s
\end{aligned}
$$


where $\alpha=1, \beta=2$ or $\alpha=2, \beta=1$,

$I_{1}(t, s)=\sum_{i=1}^{3}\left(\frac{\lambda_{i}(t)}{\lambda_{i}(s)}\right)^{2}, \quad I_{2}(t, s)=\sum_{i=1}^{3}\left(\frac{\lambda_{i}(s)}{\lambda_{i}(t)}\right)^{2}$,

$\dot{G}(s)=\mathrm{d} G(s) / \mathrm{d} s$ and $G(t) \rightarrow 0$ as $t \rightarrow \infty$. The relaxation function $G(t)$ has the form shown in Fig. 2.

The general tensorial form for the BKZ constitutive equation and the procedure for computing stresses associated with a given deformation are presented by Bernstein [7]. A detailed discussion of how (2.9) was developed was presented in [5], and will be omitted for the sake of brevity.

\section{Reduction to basic system of equations}

Using an approach introduced in a previous work on non-linear viscoelastic membranes [5], the field equations will be reduced to a more convenient form. First, let $c_{0}$ be a parameter with the dimension of stress. The latter will be suggested by a specific form for (2.8). For example, when (2.9) is used, $c_{0}=G(0)$. The following dimensionless variables are introduced: coordinates $\bar{z}=z / L_{0}, \bar{\rho}=\rho / L_{0}, \bar{\zeta}=\xi / L_{0}$, stress $\bar{\sigma}_{\alpha}=\sigma_{\alpha} / c_{0}$, length $\bar{L}=L / L_{0}$, pressure $\bar{P}=$ $p(t) a /\left(h_{0} c_{0}\right)$, and force $\bar{F}=F /\left(\pi a h_{0} c_{0}\right)$. By (2.1) and (2.2), $\bar{\lambda}_{1}=\lambda_{1}, \bar{\lambda}_{2}=\lambda_{2}$ and $\bar{\eta}=\eta$. Let $\alpha=L_{0} / a$.

Second, define the transformation

$\hat{\sigma}_{1}=\bar{\sigma}_{1} / \lambda_{1}, \quad \hat{\sigma}_{2}=\bar{\sigma}_{2} / \lambda_{2}, \quad \hat{\eta}=\eta / \lambda_{1}$.

The basic set of dependent variables consists of $\lambda_{1}, \lambda_{2}, \hat{\eta}, \hat{\sigma}_{1}, \hat{\sigma}_{2}$, for which equations are now developed. The force balance equations (2.3) and (2.4) become, using (2.5) and (3.1),

$\partial \hat{\sigma}_{1} / \partial \bar{z}=\hat{\sigma}_{2} \hat{\eta} \alpha$,

$\frac{\partial \hat{\eta}}{\partial \bar{z}}=\left[\left(1-\hat{\eta}^{2}\right) \frac{\hat{\sigma}_{2}}{\hat{\sigma}_{1}}-\bar{P} \lambda_{1} \lambda_{2} \frac{\left(1-\hat{\eta}^{2}\right)^{1 / 2}}{\hat{\sigma}_{1}}\right] \alpha$.

An alternative to (3.3) can be derived. Using (2.5), (2.7) and (3.1) and then non-dimensionalizing, the following explicit expression for $\hat{\eta}$ is obtained:

$\hat{\eta}=-\left[1-\left(\frac{\bar{F}+\bar{P} \lambda_{2}^{2}}{2 \hat{\sigma}_{1}}\right)^{2}\right]^{1 / 2}$.

The negative coefficient is chosen because of (2.2) and the assumption that the membrane will bulge out during deformation. The system of equations includes the compatibility condition

$\partial \lambda_{2} / \partial \bar{z}=\lambda_{1} \hat{\eta} \alpha$

which is derived from (2.1), (2.2) and (3.1). The system is completed by the 
two equations obtained by combining (2.8) and (3.1),

$\hat{\sigma}_{\alpha}(t)=\hat{A}_{\alpha}[\lambda(t), t]+\int_{0}^{t} \hat{B}_{\alpha}[\lambda(t), \lambda(s), t-s] \mathrm{d} s, \quad(\alpha=1,2)$.

Equations (3.2), (3.3), (3.5), (3.6) or (3.2), (3.4)-(3.6) are alternate systems which must be satisfied at each time $t$ for $0 \leqslant \bar{z} \leqslant 1$.

Boundary conditions are now stated in terms of the basic set of variables. By the assumed symmetry of deformation about the mid-plane $\bar{z}=0$, it follows from (2.2) and (3.1) that

$\hat{\eta}(0, t)=0$.

Since the radius of the material which is bonded to the end plates cannot change, (2.1) implies

$\lambda_{2}(1, t)=1$.

If the length history $\bar{L}(t)$ is prescribed, (2.1) and (3.1) give

$\bar{\zeta}(1, t)=\int_{0}^{1} \lambda_{1}\left[1-\hat{\eta}^{2}\right]^{1 / 2} \mathrm{~d} \bar{z}=\bar{L}(t)$

Alternatively, if the force history $\bar{F}(t)$ is prescribed, the variables must satisfy $\bar{F}(t)=\left(2 \hat{\sigma}_{1}-\bar{P} \lambda_{2}^{2}\right)_{\bar{z}=0}$.

In the remainder of this paper, the bars will be dropped for notational convenience.

The formal procedure for solving either of the alternate systems subject to (3.7), (3.8) and either (3.9) or (3.10) will be outlined here. The numerical details will be presented in Section 4 . Let time $t$ be fixed and suppose the past histories $\lambda_{1}(z, s), \lambda_{2}(z, s), 0 \leqslant s<t$, have been found. Then (3.6) can be regarded as equations relating $\hat{\sigma}_{1}$ or $\hat{\sigma}_{2}$ to $\lambda_{1}$ and $\lambda_{2}$ at time $t$, i.e.

$\hat{\sigma}_{\alpha}(t)=\hat{\sigma}_{\alpha}\left(\lambda_{1}(t), \lambda_{2}(t), t, z\right), \quad(\alpha=1,2)$.

The dependence of these relations on past history is denoted by $t$. The known spatial variation of the past history induces a $z$-dependence. Now, since its spatial derivative is not present in (3.2)-(3.6), $\hat{\sigma}_{2}$ can be expressed in terms of $\lambda_{1}, \lambda_{2}$ using (3.11). It is then assumed that the equation for $\hat{\sigma}_{1}$ in (3.11) can be inverted to give

$\lambda_{1}(t)=g\left[\hat{\sigma}_{1}(t), \lambda_{2}(t), t, z\right]$.

This is then used to eliminate $\lambda_{1}(t)$ from (3.2)-(3.6). System (3.2), (3.3), (3.5), (3.6) reduces to a system of ordinary differential equations at time $t$ of form

$\mathrm{d} \Lambda / \mathrm{d} z=\boldsymbol{F}(\Lambda, z, P)$, 
where $\Lambda=\left(\hat{\sigma}_{1}, \hat{\eta}, \lambda_{2}\right)^{\mathrm{T}}$. In view of (3.4), the alternate system (3.2), (3.4)(3.6) reduces further to a second-order system where $\Lambda=\left(\hat{\sigma}_{1}, \lambda_{2}\right)^{\mathrm{T}}$.

Equation (3.13) together with end conditions (3.7)-(3.10) define a two point boundary value problem at time $t$. The nature of this problem depends on whether the axial force $F(t)$ or the elongation $L(t)$ is specified along with the internal pressure $P(t)$. If the axial force is specified, system (3.2), (3.4)(3.6) can be used since (3.4) is expressed in terms of $F$ and $P$. Integration of (3.13), with $\Lambda=\left(\hat{\sigma}_{1}, \lambda_{2}\right)^{\mathrm{T}}$, requires values for $\lambda_{2}$ and $\hat{\sigma}_{1}$ at $z=0$. A value for $\hat{\sigma}_{1}(0, t)$ can be estimated and $\lambda_{2}(0, t)$ can be found from (3.10). It can be seen from (3.4) that this automatically satisfies (3.7). The estimates of $\hat{\sigma}_{1}(0, t)$ are corrected in order to satisfy $(3.8)$ by a shooting method. The elongation $L(t)$ can be computed once the solution to the boundary value problem has been obtained.

If the elongation is specified, the axial force $F$ is determined using (3.10) only after the solution has been obtained. In this case, (3.4) cannot be conveniently used and system (3.2), (3.3), (3.5), (3.6) is the one to be solved. Integration of (3.13), with $\Lambda=\left(\hat{\sigma}_{1}, \lambda_{2}, \hat{\eta}\right)^{\mathrm{T}}$, requires values for $\hat{\sigma}_{1}, \lambda_{2}$ and $\hat{\eta}$ at $z=0$. The initial value for $\hat{\eta}$ is given by (3.7). The values for $\lambda_{2}(0, t)$ and $\hat{\sigma}_{1}(0, t)$ must be estimated and then adjusted by a shooting method in order to satisfy (3.8) and (3.9) at $z=1$. Thus, if elongation is specified, a two-dimensional shooting method is needed, whereas if force is prescribed, a one-dimensional method is used.

In the remainder of this work, it is assumed that elongation is prescribed. There is an experimental and a computational reason for this choice. It is expected that an experiment could be designed so that either an axial force or an elongation could be controlled. If force is prescribed, it is hard to estimate a priori the size of the deformation or the range of values of the stretch ratios near $z=0$. On the other hand, if the elongation is specified, then $\lambda_{1}(0, t)$ is, in dimensional variables, approximately $L(t) / L_{0}$ at least initially. This allows some control over experimental conditions.

Regarding computation, the one-dimensional shooting method required in the axial force approach would be essentially the same as that used in previous work $[4,5]$. On the other hand, the elongational problem requires the development of a two-dimensional shooting method. The experience gained with this more advanced procedure could be useful in the numerical solution of problems involving other deformation configurations, e.g. as in the modeling of drape forming.

\section{Numerical procedure}

There are two classes of numerical methods which are used in the solution of the problem. The first has to do with the numerical means by which the system (3.2), (3.3), (3.5), (3.6) or the system (3.2), (3.4)-(3.6) is reduced to form (3.13) and then integrated. Since this is essentially the same as the procedure described in [5] it will be omitted for the sake of brevity. The sec- 
ond, which is the method of two-dimensional shooting used to satisfy the boundary conditions, will be discussed here.

For the system (3.2), (3.3), (3.5), (3.6), subject to boundary conditions (3.7)-(3.9), let $t_{1}=0, t_{2}, . ., t_{n-1}, t_{n}$ be a set of times and let $z_{1}=0, z_{2}, \ldots$, $z_{i}, \ldots, z_{m}=1$ be a set of space mesh points at which the solution is to be found. Then, as described in [5], (3.13) is integrated at each fixed time step $t_{n}$ from $z_{1}=0$ using (3.7) and assumed values for $\lambda_{2}\left(z_{1}, t_{n}\right)$ and $\hat{\sigma}_{1}\left(z_{1}, t_{n}\right)$. In the actual computation, it was found convenient to assume values for $\lambda_{2}\left(z_{1}, t_{n}\right)$ and $\lambda_{1}\left(z_{1}, t_{n}\right)$, and compute $\hat{\sigma}_{1}\left(z_{1}, t_{n}\right)$. This avoided the numerical inversion of (3.11) at the first node. Introduce the notation $\lambda_{1}\left(z_{1}, t_{n}\right)=x_{1}, \lambda_{2}\left(z_{1}, t_{n}\right)=$ $x_{2}$. The end values $\lambda_{2}\left(1, t_{n}\right)$ and $\zeta\left(1, t_{n}\right)$ can be regarded as functions of $x_{1}$ and $x_{2}$,

$\lambda_{2}\left(1, t_{n}\right)=\tilde{\lambda}\left(x_{1}, x_{2}\right)$,

$\zeta\left(1, t_{n}\right)=\tilde{\zeta}\left(x_{1}, x_{2}\right)$.

An explicit form for these functions cannot generally be found. They are known only through the values of $\lambda_{2}\left(1, t_{n}\right)$ and $\zeta\left(1, t_{n}\right)$ obtained for the various choices of the initial conditions $x_{1}, x_{2}$ arising during iteration. The proper choice of the initial conditions $x_{1}^{*}, x_{2}^{*}$, by (4.1), (3.8) and (3.9), satisfies

$\tilde{\lambda}\left(x_{1}^{*}, x_{2}^{*}\right)=1$

$\tilde{\zeta}\left(x_{1}^{*}, x_{2}^{*}\right)=L\left(t_{n}\right) \equiv L_{n}$.

The solution of this system is obtained by Newton's method. Let $x_{1}^{(i)}, x_{2}^{(i)}$ be the results of the $i$ th iteration. Then the corrections $\Delta x_{1}, \Delta x_{2}$ are found by solving

$\frac{\partial \tilde{\lambda}^{(l)}}{\partial x_{1}} \Delta x_{1}+\frac{\partial \tilde{\lambda}^{(i)}}{\partial x_{2}} \Delta x_{2}=1-\tilde{\lambda}\left(x_{1}^{(i)}, x_{2}^{(i)}\right)$,

$\frac{\partial \tilde{\zeta}^{(i)}}{\partial x_{1}} \Delta x_{1}+\frac{\partial \tilde{\zeta}^{(i)}}{\partial x_{2}} \Delta x_{2}=L_{n}-\tilde{\zeta}\left(x_{1}^{(i)}, x_{2}^{(i)}\right)$,

where the superscript on the partial derivatives indicates that they are to be evaluated at $\left(x_{1}^{(i)}, x_{2}^{(i)}\right)$.

The derivatives are up-dated using data from the $i$ th, $(i-1)$ st, $(i-2)$ nd iterations, referred to as points A, B, C, respectively, in Fig. 3. Expanding $\tilde{\lambda}\left(x_{1}, x_{2}\right)$ in a Taylor series about point $A$, retaining terms in the first derivatives, and evaluating at $B$ and $C$ gives

$\left.\frac{\partial \tilde{\lambda}}{\partial x_{1}}\right|_{\mathrm{A}}\left(x_{1}^{\mathrm{C}}-x_{1}^{\mathrm{A}}\right)+\left.\frac{\partial \tilde{\lambda}}{\partial x_{2}}\right|_{\mathrm{A}}\left(x_{2}^{\mathrm{C}}-x_{2}^{\mathrm{A}}\right)=\tilde{\lambda}^{\mathrm{C}}-\tilde{\lambda}^{\mathrm{A}}$,
$\left.\frac{\partial \tilde{\lambda}}{\partial x_{1}}\right|_{\mathrm{A}}\left(x_{1}^{\mathrm{B}}-x_{1}^{\mathrm{A}}\right)+\left.\frac{\partial \tilde{\lambda}}{\partial x_{2}}\right|_{\mathrm{A}}\left(x_{2}^{\mathrm{B}}-x_{2}^{\mathrm{A}}\right)=\tilde{\lambda}^{\mathrm{B}}-\tilde{\lambda}^{\mathrm{A}}$.

Solving this system gives the partial derivatives for use in the first of (4.3). 


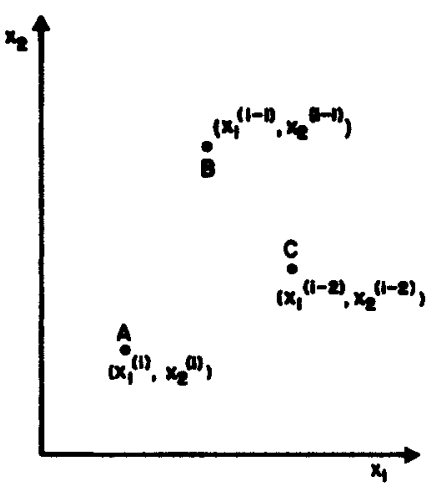

Fig. 3. Points A, B, C in the method for estimating derivatives using (4.7).

Repeating this for $\tilde{\zeta}\left(x_{1}, x_{2}\right)$ gives the partial derivatives for use in the second of (4.3). The procedure is initiated with Points A, B, C defined at $\left(x_{1}^{0}, x_{2}^{0}\right)$, $\left(x^{0}(1+\epsilon), x_{2}^{0}\right),\left(x_{1}^{0}, x_{2}^{0}(1+\epsilon)\right)$. The boundary conditions are considered satisfied when the sum of the squares of the right-hand sides of (4.3) is less than some tolerance.

An initial estimate $x_{1}^{0}$ and $x_{2}^{0}$ must be made at each time. At $t_{1}=0$, the membrane is undeformed and $x_{1}=x_{2}=1.0$. At $t_{2}, x_{1}^{0}$ and $x_{2}^{0}$ are specified as input data. For later times, $x_{1}^{0}$ and $x_{2}^{0}$ are determined by extrapolation from converged values at previous times. At $t_{3}$, the extrapolation is linear. For $t_{i}>t_{3}$, it is quadratic.

\section{Numerical example and discussion}

For the purposes of a numerical example, the membrane is regarded as a viscoelastic fluid whose constitutive equation is given by (2.9). The method for determining an expression to fit the relaxation function shown in Fig. 2 is discussed in [5]. This reference also contains a discussion of the method used to select the times $t_{i}$ at which the solution is to be obtained.

In the numerical example presented here, $L_{0} / a=1$. The pressure history is given by $P(t)=0.25 t, t \leqslant 9.36, P(t)=2.34, t \geqslant 9.36$. The elongation history is given by $L(t) / L_{0}=1+0.01 t$. This causes the length to double after 100 seconds. These histories along with the computed axial force history are shown in Fig. 4.

This rate of pressure increase was selected because at lower rates the membrane would draw in. The pressure was held constant for $t \geqslant 9.36$ for reasons motivated by previous studies. In the elastic membrane version of the problem treated here [8] the following was found. For a fixed elongation, the internal pressure increases with the radius at $z=0$, the maximum radius, to some maximum value and then decreases. If the pressure becomes greater than this maximum or limiting value, either an equilibrium solution is not pos- 


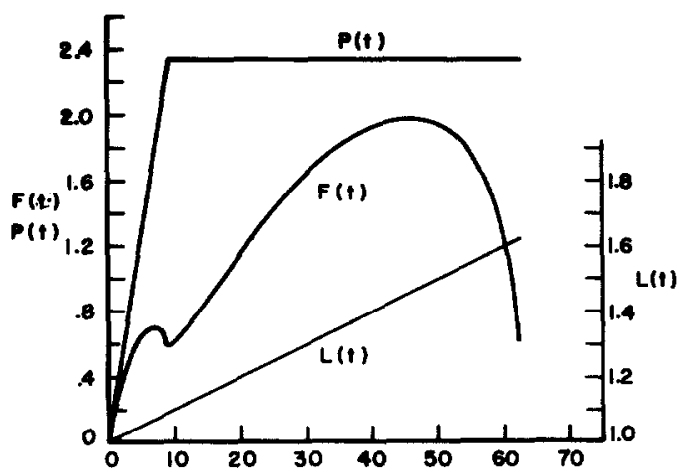

Fig. 4. Prescribed length history $L(t)$, pressure history $P(t)$ and computed axial force history $F(t)$.

sible or the radius has a jump discontinuity. The limit pressure decreases with increasing elongation. In another study involving the inflation of a viscoelastic spherical membrane [9], a similar situation is shown to occur. There is a limit pressure at each time. If the actual pressure is below this limit at time $t$, a continuous deformation history is possible. This limit pressure decreases with time as the sphere inflates in a manner which depends on deformation history. For pressures in the neighborhood of the limit pressure, the radius increases very rapidly. In the present problem, the combination of viscoelasticity and increasing elongation could cause a rapid decrease in the value of the limit pressure. For a monotonically increasing pressure history, the limit pressure could be exceeded at a very early time. Preliminary results using such a pressure history suggested this might be occurring. A determination of the limit pressure history, the time when it might be exceeded by a given pressure history and the deformation history, as in [9], would be useful. However, the computation is very difficult and should be the subject of a separate study. It was felt that these difficulties would be avoided or delayed for the pressure history specified above.

The deformed profiles at several times and the particle paths are shown in Fig. 5. The stretch ratio histories at the midplane, $z=0$, are shown in Fig. 6 . Recalling (2.1), the graph for $\lambda_{2}$ also represents the midplane radius history. The stress histories at $z=0$ are shown in Fig. 7. The distributions of the stretch ratios and stress with respect to the reference coordinate are shown in Figs. 8 and 9.

Figures 6 and 8 show that the distribution of $\lambda_{1}$ can be considered uniform for $t<50$. This implies that within this time range the approximation $\lambda_{1}(t) \approx$ $L(t) / L_{0}$ is reasonable. Figure 6 also shows that the midplane $\lambda_{2}$ history increases rapidly at first until $P(t)$ becomes constant, after which it increases slowly. Then at about $t=50$, both $\lambda_{1}$ and $\lambda_{2}$ begin to increase very rapidly. In other terms, the radial inflation of the tube accelerates, indicating, perhaps, the onset of an instability. Results were not obtained for $t>62.0$. At the 


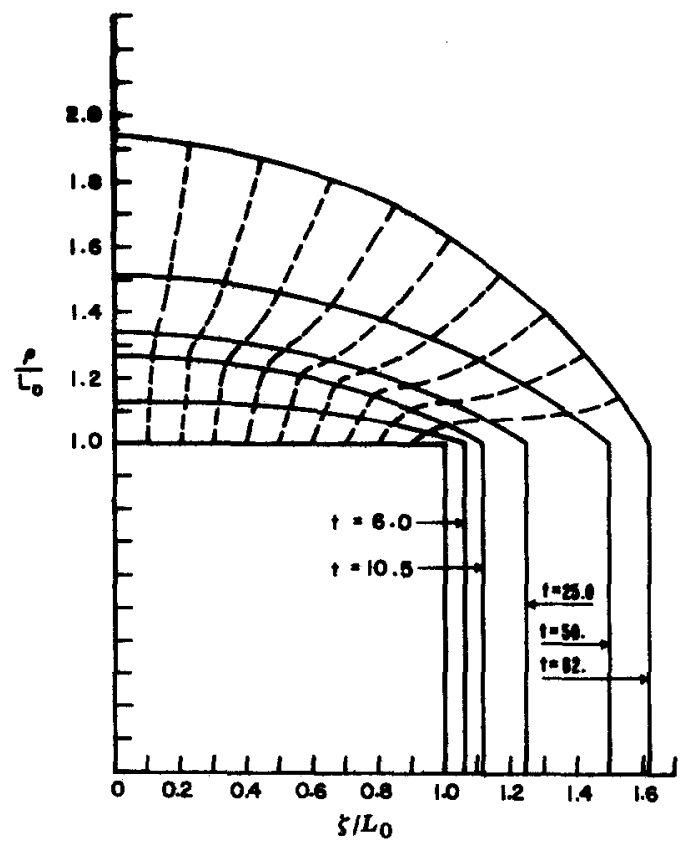

Fig. 5. Deformed profiles at several times. The dashed lines represent particle paths.
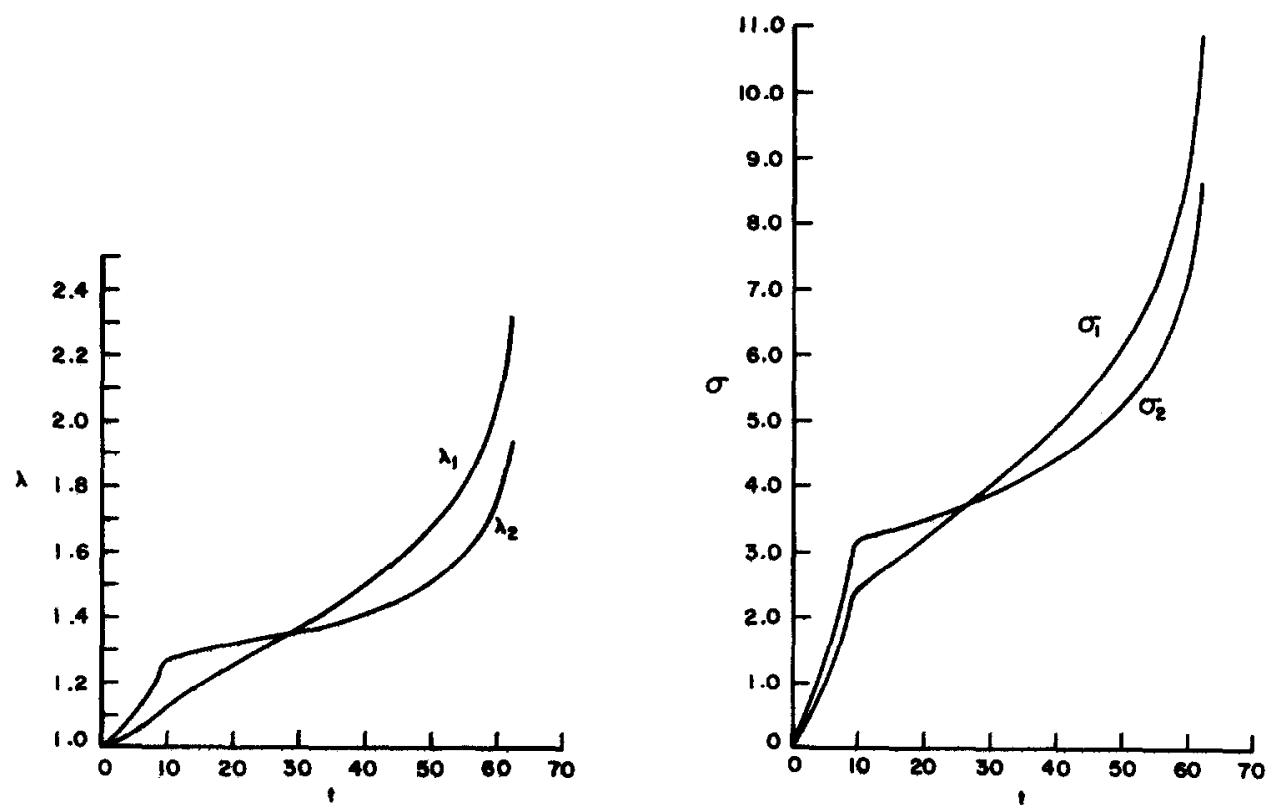

Fig. 6. Histories of principal stretch ratios at $z=0$. The $\lambda_{2}$ graph also denotes the dimensionless radius history.

Fig. 7. Histories of principal stresses at $z=0$. 

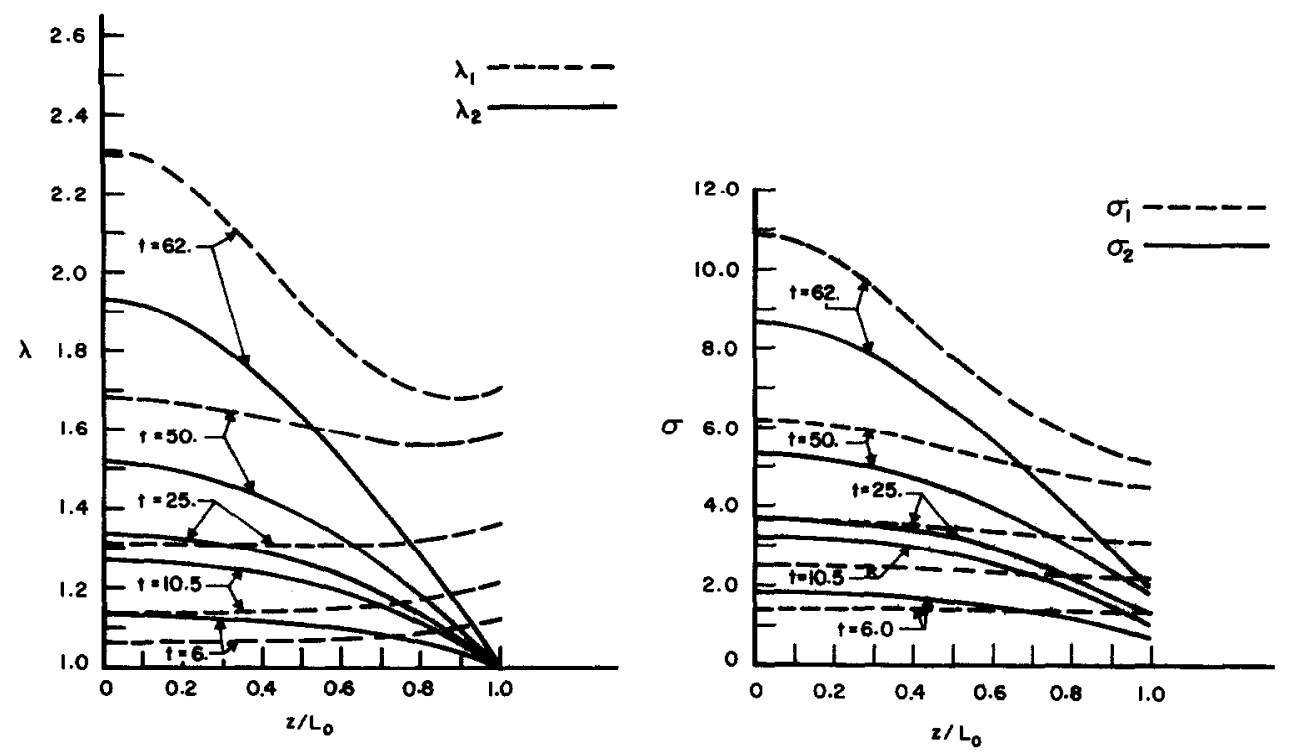

Fig. 8. Distribution of principal stretch ratios with respect to the reference coordinate.

Fig. 9. Distribution of principal stresses with respect to the reference coordinate.

next solution time, numerical difficulties arose and computation ceased. This will be discussed further below.

This behavior suggests that the earlier discussion applies here. There appears to be a limit pressure history whose value is decreasing. As this value approaches $P=2.34, \mathrm{~d} \lambda_{2}(0, t) / \mathrm{d} t \rightarrow \infty$, which is consistent with the results in [9]. If the limit pressure becomes less than $P=2.34$, a continuous equilibrium solution is not possible. This suggests further that an instability is developing.

The axial force history $F(t)$, shown in Fig. 4, is not a strictly monotonically increasing function as might have been expected. It increases until $t=7.11$ and then decreases while the pressure is still increasing. When the pressure is held constant at $t=9.36, F(t)$ again increases until $t=46.4$ when it begins to decrease a second time. These decreases are apparently not due to stress relaxation, since the stresses at the midplane increase monotonically, as seen in Fig. 7. This behavior can be explained using (3.10), which expresses the balance of forces acting on the portion of the tube to one side of the midplane $z=0$. Let $F_{T}$ and $F_{P}$ denote the first and second terms in (3.10). $F_{T}$ represents the tensile force on the annular cross-section of the tube material at $z=0$, equal to the product of the stress and the current material crosssectional area. $F_{P}$ represents the resultant force due to the internal pressure, equal to the product of the pressure and the area enclosed by the circumference. Since $F_{P}$ acts in the same sense as the externally applied axial force, the negative sign is needed. As $t$ increases, $F_{r}$ increases. A greater portion of 
$F_{T}$ becomes balanced by the increasing pressure acting on the increasing enclosed area. This results in a decrease in the externally applied axial force required for equilibrium. When the pressure is held constant, the radial tube expansion is slowed down, as indicated by the graph of $\lambda_{2}$ for $10 \leqslant t \leqslant 40$ in Fig. 6. $F_{P}$ increases more slowly and the axial force must increase in order to maintain equilibrium. For $t>40$, the radius and hence $F_{P}$ begin to increase rapidly. As this balances a larger portion of $F_{T}$, the axial force once more decreases.

The evaluation of the inverse (3.12) during the integration of the system (3.2), (3.3), (3.5), (3.6) caused no numerical difficulties. The iteration procedure for satisfying the boundary conditions worked reasonably well. Solutions were obtained at 57 times for this numerical example. The convergence tolerance was $10^{-8}$. The problem of satisfying the boundary conditions appeared to be quite sensitive to the initial estimate $\left(x_{1}^{0}, x_{2}^{0}\right)$ at the earlier times. At $t_{2}$, this estimate was determined by trial and error. If $\left(x_{1}^{0}, x_{2}^{0}\right)$ was not sufficiently close to the required value, the argument of the square root in (3.3) became negative and computation ceased. Choices for $\left(x_{1}^{0}, x_{2}^{0}\right)$ at later times were determined automatically by extrapolation. Twenty iterations were required at $t_{3}, 27$ at $t_{4}$ and 15 at $t_{5}$. The number of iterations then decreased. Generally, between $t_{8}=7.11$ and $t_{46}=49.75$ at most 10 iterations were required. Then from $t_{47}=50.87$ to $t_{57}=62.1$, as the deformation began to increase rapidly, 12-14 iterations were needed. In the long middle interval, $\left(x_{1}^{0}, x_{2}^{0}\right)$ was very close to the accepted iterate. This can be attributed to the decreased sensitivity of the problem to the initial estimate, to the smoothly varying $\lambda_{1}$ and $\lambda_{2}$ histories, as shown in Fig. 6, and to the quadratic extrapolation. At the last time, $t_{58}$, one of the values $x_{\alpha}^{(n)}$ became negative, which is physically unreasonable. The above-mentioned square root problem arose and computation ceased. It is not clear whether this is a consequence of the approximations involved in (4.3) and (4.4) or whether it reflects numerical difficulties arising from the rapid rate of increase of $\lambda_{1}$ and $\lambda_{2}$.

For values of $L_{0} / a>1$, results for only a few steps were obtained. If at some step $t_{n}$ an iterate $\left(x_{1}^{(i)}, x_{2}^{(i)}\right)$ was not sufficiently close to the solution of (4.5), the argument of the square root in (3.3) became negative and computation ceased. This was caused by either (1) a poor initial estimate $\left(x_{1}^{0}, x_{2}^{0}\right)$ obtained by extrapolation or (2) a large change in a subsequent iterate.

It was found that functions $\tilde{\lambda}$ and $\widetilde{\zeta}$ in (4.2) varied rapidly with their arguments. This indicates that the extrapolation problem (1) appears to be a consequence of the high sensitivity of the solution to the choice of $\left(x_{1}^{0}, x_{2}^{0}\right)$ at early times. The iteration problem (2) appears to be caused by the numerical error in approximating the derivatives using (4.4) and the possible poor conditionality of (4.3).

For future work, it would be desirable to develop a method for correcting an iterate in the event either (1) or (2) occurs. The simplest general two-dimen. sional correction scheme appears to involve the modification of the computer program to allow operator-computer interaction. When an iterate leads to a 
stoppage of computation at some time $t_{n}$, the operator would specify a new choice for $\left(x_{1}^{0}, x_{2}^{0}\right)$ and computation would resume. The choices would be based on experience gained by trial and error.

In conclusion, it appears that the method presented here is adequate for the simulation of the tube inflation-extension experiment under certain conditions. With the inclusion of the modifications discussed above, this method should become more generally useful.

\section{Acknowledgements}

The author expresses his gratitude to Robert Benedict for computing assistance, to the National Bureau of Standards, and to the National Science Foundation for their support of this work under Grant No. ENG 75-17489.

\section{References}

1 A.E. Green and J.E. Adkins, Large Elastic Deformations and Nonlinear Continuum Mechanics, Oxford University Press, 1960.

2 D.D. Joye, G.W. Poehlein and C.D. Denson, Trans. Soc. Rheol., 16 (1972) 421.

3 S.C.-K. Chung and J.F. Stevenson, Rheol. Acta, 14 (1975) 832.

4 A.S. Wineman, Trans. Soc. Rheol., 20 (1976) 203.

5 A.S. Wineman, J. Non-Newtonian Fluid Mech., 4 (1978) 249.

6 B. Bernstein, E.A. Kearsley and L.J. Zapas, Trans. Soc. Rheol., 7 (1963) 391.

7 B. Bernstein, Acta Mech., 11 (1966) 329.

8 Robert Benedict, Alan Wineman and Wei H. Yang, Int. J. Solids Struct., to appear.

9 Alan Wineman, Int. J. Solids Struct., 14 (1978) 197. 